\title{
High-efficiency tunable T-shaped beam splitter based on one-way waveguide
}

\author{
Hong, Lujun; Xiao, Sanshui ; Deng, Xiaohua; Pu, Rongqiang; Shen, Linfang
}

Published in:

Journal of Optics

Link to article, DOI:

$10.1088 / 2040-8986 /$ aaeaac

Publication date:

2018

Document Version

Peer reviewed version

Link back to DTU Orbit

Citation (APA):

Hong, L., Xiao, S., Deng, X., Pu, R., \& Shen, L. (2018). High-efficiency tunable T-shaped beam splitter based on one-way waveguide. Journal of Optics, 20(12), [125002]. https://doi.org/10.1088/2040-8986/aaeaac

\section{General rights}

Copyright and moral rights for the publications made accessible in the public portal are retained by the authors and/or other copyright owners and it is a condition of accessing publications that users recognise and abide by the legal requirements associated with these rights.

- Users may download and print one copy of any publication from the public portal for the purpose of private study or research.

- You may not further distribute the material or use it for any profit-making activity or commercial gain

- You may freely distribute the URL identifying the publication in the public portal

If you believe that this document breaches copyright please contact us providing details, and we will remove access to the work immediately and investigate your claim. 


\title{
High-efficiency tunable T-shaped beam splitter based on one-way waveguide
}

\author{
Lujun Hong ${ }^{1,2,3}$, Sanshui Xiao ${ }^{2}$, Xiaohua Deng ${ }^{3}$, \\ Rongqiang $\mathbf{P u}^{4}$, and Linfang Shen ${ }^{3,5}$ \\ ${ }^{1}$ Key laboratory of Poyang Lake Environment and Resource Utilization of Ministry \\ of Education, School of Resources Environmental and Chemical Engineering, \\ Nanchang University, Nanchang 330031, China \\ 2 DTU Fotonik, Department of Photonics Engineering, Technical University of \\ Denmark, DK-2800 Kgs. Lyngby, Denmark \\ ${ }^{3}$ Institute of Space Science and Technology, Nanchang University, Nanchang 330031, \\ China \\ ${ }^{4}$ Department of Information Engineering, Wuhu Institute of Technology, Wuhu \\ 241000, China \\ ${ }^{5}$ Department of Applied Physics, Zhejiang University of Technology, Hangzhou \\ 310023, China \\ E-mail: saxi@fotonik.dtu.dk, lfshen@ncu.edu.cn
}

\begin{abstract}
Power splitters have been widely studied in the fields of radio frequency and integrated photonics, however, conventional splitters generally suffer with poor transmission and limited bandwidth. Here we propose a novel tunable T-shaped power splitter utilizing one-way waveguide that is composed by the yttrium-iron-garnet (YIG)-air-metal configuration with one static magnetic field applied. We show that the transmission efficiency of such a splitter reaches $100 \%$ in the lossless case due to the suppression of reflection by the one-way waveguide. By introducing a defect in the junction area, the splitting ratio of the splitter can be gradually tuned by varying the external magnetic field, and large operation bandwidth can be achieved for a given splitting ratio. Our results presented here focus on the radio frequency, and the concept can easily be applied to the photonic area.
\end{abstract}

Keywords: Power splitter, one-way propagation, surface plasmons, waveguide 


\section{Introduction}

Power splitter is one of the key elements in radio technology [1, 2, 3] and integrated photonics $[4,5,6,7]$, and has been widely used for many applications including interferometers [8], optical switches [9, 10], and multiplexing devices [11, 12]. T-splitters and Y-splitters are the commonly used power splitters $[13,14,15,16]$. For these splitters, two fundamental issues of their design are tunable splitting ratio and high transmission efficiency. Having multimodes in the junction often leads to mode-mixing problems, giving the rise to the mode-match problem with respect to the input and output modes [15]. Therefore, significant reflection at the junction is normally observed. To improve the transmission of the splitters, lots of work have been performed by developing better junctions [17, 18]. For a unequal power splitter, the modification of the junction structure naturally results in the variation of the splitting ratio, however giving rise to the big challenge for the design of tunable devices.

Recently, many groups have proposed an alternative and effective way to improve the transmission efficiency of splitters by using one-way electromagnetic (EM) waveguide $[19,20]$, which was firstly proposed as analogues of quantum Hall edge states in photonic crystals (PhCs) $[21,22,23]$. The mode supported by the one-way EM waveguide can only propagate in one direction, and is immune to backscattering at discontinuities or bends because of the absence of a back-propagating mode [24, 25, 26, 27, 28, 29]. The one-way waveguide enables us to deal with the issue of the transmission efficiency [30] in the design of the power splitter. Based on a gyromagnetic material, i.e., yttrium-irongarnet (YIG), different PhC T-splitters incorporating the concept of one-way waveguide have been proposed at microwave frequencies. It has been shown that the transmission efficiency is always $100 \%$ in the lossless case [19, 20]. Moreover, these splitters exhibit high-efficiency and reflection-free even with obstacles, which would be impossible for the regular $\mathrm{PhC}$ splitters studied before. However, in those devices, two or more uniform dc magnetic fields with different directions are required. More recently, we have reported a robust one-way waveguide formed by bulk gyromagnetic YIG [31]. This system supports not only one-way surface magnetoplasmons (SMPs) but one-way regular modes, which are confined by total internal reflection. These one-way regular modes can also be immune to backscattering. Compared to one-way PhC modes, the one-way waveguide we proposed in Ref. [31] is simple in geometry and the design is more robust in mechanism.

In this paper, we propose a tunable T-shaped splitter, where the input and output waveguides are composed by our proposed one-way waveguide i.e., YIG-air-metal configuration with one static magnetic field applied. Here only one external magnetic field is needed. We show that the device possesses high efficiency and its tunable splitting ratio by varying the external magnetic field when a defect is introduced in the junction of the splitter. Finally broad-band operation with high-efficiency is also presented. 


\section{Dispersion Property of One-way Waveguide}

The one-way waveguide is formed by the YIG-air-metal configuration, see the inset of Fig. 1(b), where the thickness of the air layer is denoted by $d$, and where the thickness of the metal/YIG is semi-infinite. The magnetized YIG is gyromagnetic anisotropic with permittivity $\varepsilon_{m}(=15)$ and permeability tensor $\mu_{m}$, which takes the form [32]

$$
\overleftrightarrow{\mu}_{m}=\left[\begin{array}{ccc}
\mu_{1} & -i \mu_{2} & 0 \\
i \mu_{2} & \mu_{1} & 0 \\
0 & 0 & 1
\end{array}\right]
$$

with

$$
\begin{aligned}
& \mu_{1}=1+\frac{\omega_{m}\left(\omega_{0}-i v \omega\right)}{\left(\omega_{0}-i v \omega\right)^{2}-\omega^{2}}, \\
& \mu_{2}=\frac{\omega_{m} \omega}{\left(\omega_{0}-i v \omega\right)^{2}-\omega^{2}},
\end{aligned}
$$

where $\omega$ is the angular frequency, $\omega_{m}$ is the characteristic circular frequency, $v$ is the damping coefficient, and $\omega_{0}=2 \pi \gamma H_{0}$ ( $\gamma$ is the gyromagnetic ratio) is the precession angular frequency. Such a three-layer structure can support both SMPs and regular modes, whose dispersion relations are given by [31]

$$
\begin{aligned}
& \alpha_{r} \mu_{v}+\left(\alpha_{m}+\frac{\mu_{2}}{\mu_{1}} k\right) \tanh \left(\alpha_{r} d\right)=0, \quad \text { SMP } \\
& p \mu_{v}+\left(\alpha_{m}+\frac{\mu_{2}}{\mu_{1}} k\right) \tan (p d)=0, \quad \text { regular mode, }
\end{aligned}
$$

where $k$ is the propagation constant, $\alpha_{m}=\sqrt{k^{2}-\varepsilon_{m} \mu_{v} k_{0}^{2}}$ with $\mu_{v}=\mu_{1}-\mu_{2}^{2} / \mu_{1}$ (the Voigt permeability), $p=\sqrt{{k_{0}{ }^{2}-k^{2}}^{2}}$, and $\alpha_{r}=\sqrt{k^{2}-k_{0}{ }^{2}}$ with $k_{0}=\omega / c$ being the vacuum wavenumber. The linear terms of $k$ in Eqs. (2) and (3) indicate that both SMPs and regular modes supported by the YIG-air-metal structure are nonreciprocal. Figure 1(a) shows the dispersion relations for the regular mode (the red solid line) and SMPs (the blue dashed lines), which lie up and below of the light cone (the black dot-dashed lines), respectively. Here we assume the YIG medium to be lossless $(v=0)$ with $\varepsilon_{m}=15$ and $\omega_{m}=10 \pi \times 10^{9} \mathrm{rad} / \mathrm{s}\left(f_{m}=5 \mathrm{GHz}\right)$, and $\omega_{0}$ is set at $0.5 \omega_{m}$, which is equivalent to $\mathrm{H}_{0}=893 \mathrm{G}$. In this work, the thickness of the air layer is fixed as $d=0.05 \lambda_{m}$, i.e., $d=3 \mathrm{~mm}$. In Ref. [31], we have shown that the dispersion relation and bandwidth for the one-way propagation are affected by the thickness of the dielectric layer $d$. Here we choose $d=3 \mathrm{~mm}$ which provides the largest bandwidth. The bands of two types of modes are connected at the light lines $(\omega=c|k|)$, as shown in Fig. 1(a). The uppermost and lowest shaded areas represent zones of bulk modes $\left(k^{2}<\varepsilon_{m} \mu_{v} k_{0}{ }^{2}\right)$ supported by the YIG, whose band gap ranges from $\omega_{a}=\sqrt{\omega_{0}\left(\omega_{0}+\omega_{m}\right)}$ to $\omega_{b}=\omega_{0}+\omega_{m}$. The middle shaded area represents the bandwidth exhibiting robust one-way propagation (ROWP). The lower limit of ROWP is equal to $\omega_{s p}=\omega_{0}+\omega_{m} / 2$, i.e., the asymptotic frequency of SMP when $k \rightarrow-\infty$, and the upper limit is $\omega_{b}$ associated with the lower limit of the second bulk-mode zone in YIG. As seen in Fig. 1(a), the ROWP band ranges from $\omega_{m}$ to 

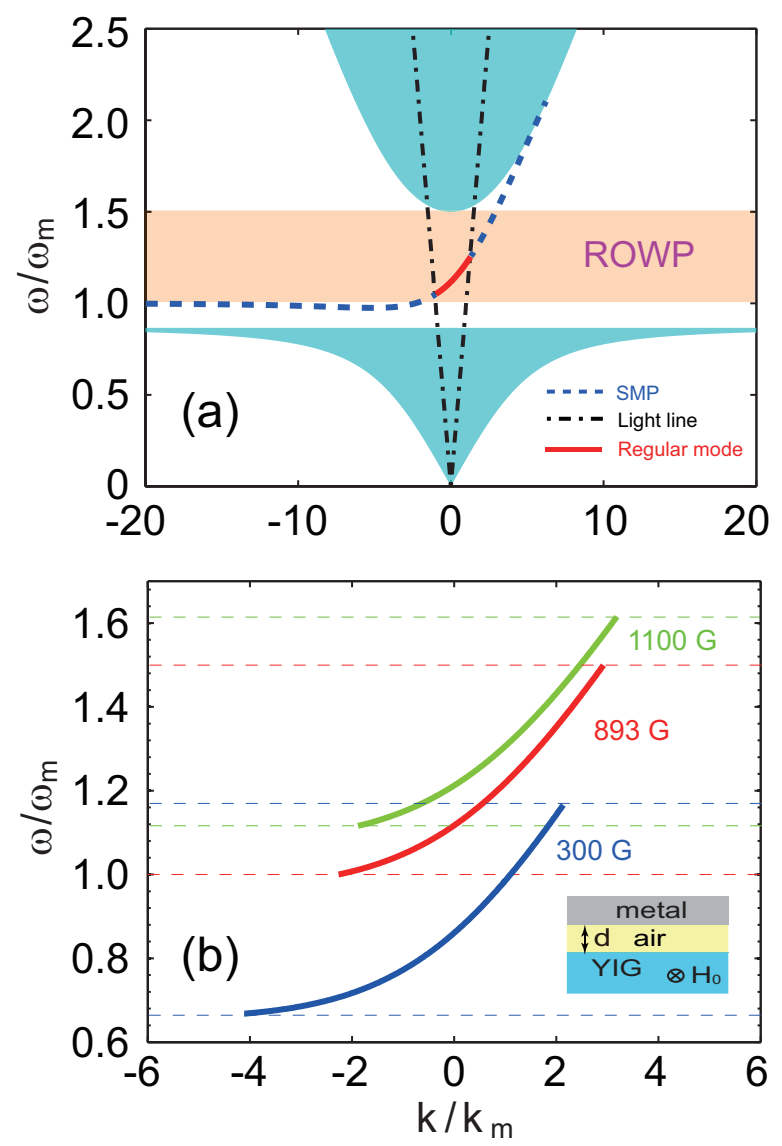

Figure 1. (Color online)(a) Dispersion relations of SMPs and regular mode for $\mathrm{H}_{0}=893 \mathrm{G}$ in the YIG-air-metal structure. The uppermost and lowest shaded areas represent the band structure of bulk modes in the YIG, and the middle one represents the ROWP region for the structure. (b) ROWP bands for various $\mathrm{H}_{0}$ values. The solid lines from the top to bottom represent $\mathrm{H}_{0}=1100,893$, and $300 \mathrm{G}$, respectively. The inset shows the schematic of the YIG-air-metal structure, where the thickness of air layer is denoted as $d=3 \mathrm{~mm}$.

$1.5 \omega_{m}$, equivalent to the frequency region $[5,7.5] \mathrm{GHz}$. In this window, both SMPs and the regular mode are allowed to propagate only in one direction, and they are immune to backscattering at imperfects because of the absence of a back-propagating mode in the system.

The influence of the external magnetic field $\mathrm{H}_{0}$ on the ROWP band is further investigated, see Fig. 1(b). As mentioned above, the ROWP band ranges from $\omega_{s p}=\omega_{0}+\omega_{m} / 2$ to $\omega_{b}=\omega_{0}+\omega_{m}$, and the bandwidth for the ROWP is governed by $\Delta \omega=\omega_{m} / 2$, showing independence to the external magnetic field. However, both the lower and upper limit for the ROWP band shift up when increasing of $\mathrm{H}_{0}$. As an example, we analyze different $\mathrm{H}_{0}$ values: $\mathrm{H}_{0}=1100$, 893, and $300 \mathrm{G}$, see the solid lines from top to bottom in Fig. 1(b). For a given frequency, the propagation constant $(k)$ of the one-way mode changes as a function of $\mathrm{H}_{0}$, indicating that the guiding property of the one-way mode is strongly affected by the external magnetic field. 


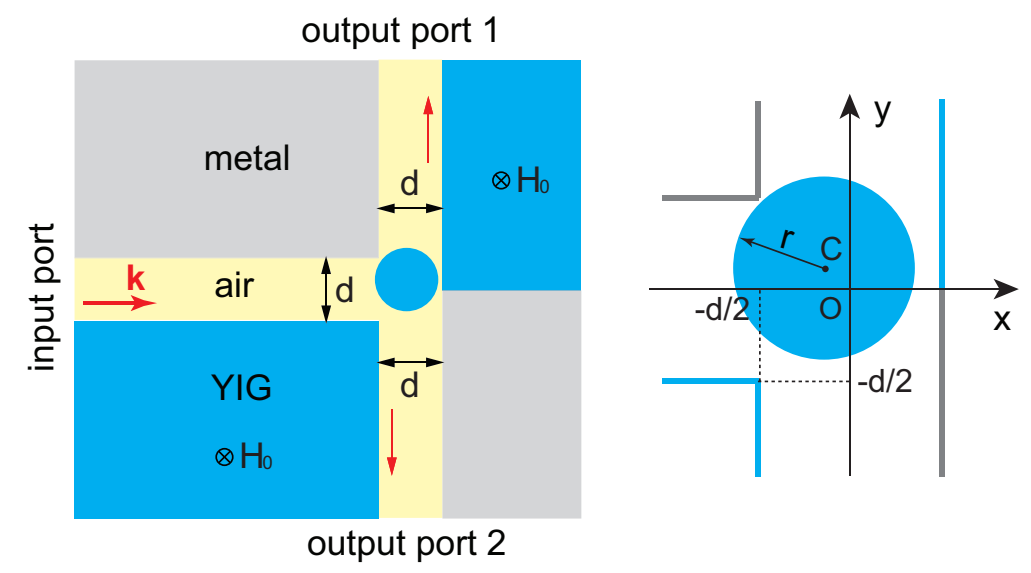

Figure 2. (Color online) Schematic of tunable T-shaped power splitter, where the YIG rod (with radius of $r$ ) in the junction region is used to tune the splitting ratio.

\section{Characteristic of the high-efficiency splitter}

The proposed T-shaped splitter based on one-way waveguides is illustrated in Fig. 2, where the input and output channels are all composed by the YIG-air-metal waveguide. For simplicity, here we consider the device in the two dimensional case. In the ROWP range of the YIG-air-metal waveguide, the input channel supports no back-propagating mode, thus incident wave can be completely split into the output channels through the junction region. Therefore, such a splitter has a perfect transmission efficiency of $100 \%$ provided that the materials in the system are all lossless. However, due to the asymmetric configuration for two output channels, the splitting ratio is very limited if we do not introduce a defect in the conjunction region. To efficiently tune the splitting ratio between the two output channels, a YIG rod with radius of $r$ is inserted in the junction region, where the center of the YIG rod is denoted by $\mathrm{C}\left(x_{c}, y_{c}\right)$. When introducing the defect, the asymmetric configuration for two output channels makes it feasible to tune the splitting ratio by varying the external magnetic field.

To verify the tunability of the proposed splitter, we perform full-wave simulations with the aid of the commercially available finite element method (COMSOL Multiphysics), and we first investigate the splitting ratio of the device without introducing any defect in the junction, as seen in Fig. 3(b). To excite waves in the input channel, a linear electric current is placed at $1.5 \mathrm{~mm}$ above the YIG surface. Due to the nature of the one-way waveguide, the incident wave from the input channel can be totally coupled into the output channels. However, the asymmetric configuration for two output channels leads to very small energy of 0.031 in the output port 1, see Fig. 3(b), when $\mathrm{H}_{0}=893 \mathrm{G}$ and $f=5.6 \mathrm{GHz}$. Our analysis also shows that the tunability as a function of the magnetic field for the structure without having the defect is also limited. We further explore the transmission properties of the power splitter when introducing the defect, as seen in Fig. 2. Note that the performance of the power splitter is strongly dependent on the size, position of YIG rod, and external applied magnetic field. We have 

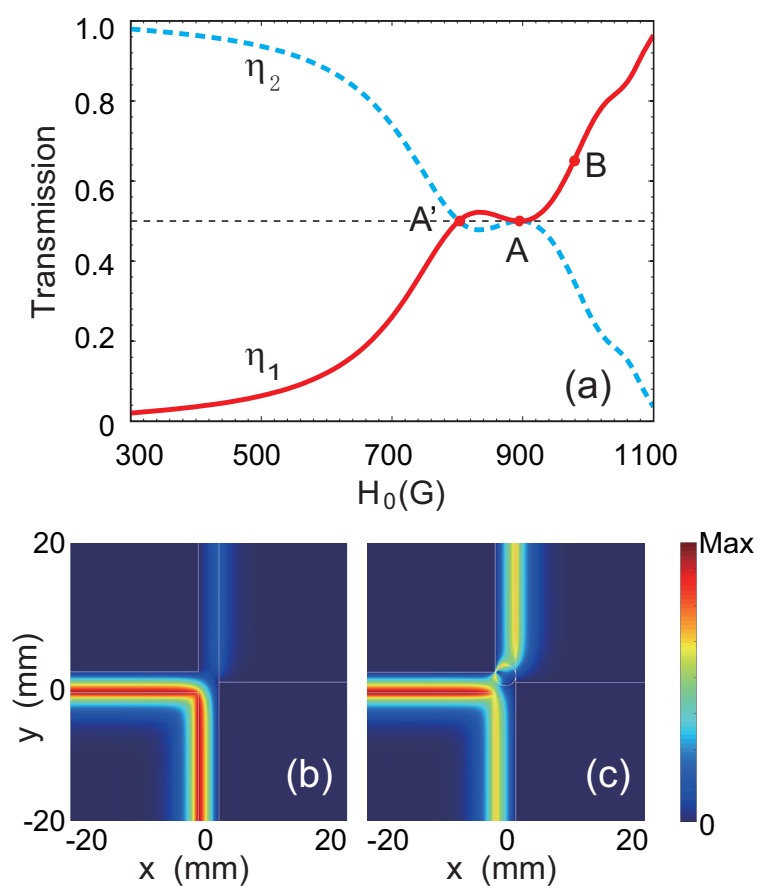

Figure 3. (Color online)(a) Transmission coefficients of the splitter assisted with the YIG rod as a function of $\mathrm{H}_{0}$. Simulated $\mathbf{E}$ amplitudes in the T-shaped power splitter without (b) and with (c) the YIG rod with the radius of $1.5 \mathrm{~mm}$ when $\mathrm{H}_{0}=893 \mathrm{G}$ and $f=5.6 \mathrm{GHz}$.

systemically analyzed the property of the proposed device, and here we choose $r=d / 2$, $x_{c}=0 \mathrm{~mm}$ and $y_{c}=1.03 \mathrm{~mm}$ as an example in order to achieve large bandwidth. The operation frequency is set to be $f=5.6 \mathrm{GHz}$, at which the input and output channels exhibit ROWP behaviour with magnetic field $\mathrm{H}_{0}$ ranging from $300 \mathrm{G}$ to $1100 \mathrm{G}$. Figure 3 (a) illustrates the the transmission coefficients $\eta_{1}\left(\eta_{2}\right)$, defined by the ratio of powers in the upper (lower) output channel to the input channel, when varying the magnetic field. With neglecting the loss of the materials, the total transmission $\left(\eta_{1}+\eta_{2}\right)$ is always unity due to ROWP feature. When introducing the defect, the transmission coefficients at two output channels can be strongly modified, by varying the magnetic field. As an example, $\eta_{1}=0.02$ and $\eta_{2}=0.98$ when $\mathrm{H}_{0}=300 \mathrm{G}$; whereas $\eta_{1}=0.96$ and $\eta_{2}=0.04$ for $\mathrm{H}_{0}=1100 \mathrm{G}$. One can find in Fig. $3(\mathrm{a})$ that $\eta_{1}$ monotonously increases with $\mathrm{H}_{0}$ except in the small interval $[802,893] \mathrm{G}$. In this interval, there always exists a pair of $\mathrm{H}_{0}$ values at which $\eta_{1}$ (as well as $\eta_{2}$ ) are the same. For example, $\eta_{1}=\eta_{2}=0.5$ at both $\mathrm{H}_{0}=893 \mathrm{G}$ and $\mathrm{H}_{0}=802 \mathrm{G}$, which are marked by $\mathrm{A}$ and $\mathrm{A}^{\prime}$ in the figure. It is found that $\eta_{1}=0.65$ and $\eta_{2}=0.35$ when $\mathrm{H}_{0}=979 \mathrm{G}$ (marked by $\mathrm{B}$ ). Figure $3(\mathrm{c}$ ) show the simulated electric field amplitudes for the cases of $\mathrm{H}_{0}=893 \mathrm{G}$, where the incident wave is equally coupled into the output channels with the aid of the defect, i.e., $\eta_{1}=\eta_{2}=0.5$. Note that the mode presented here refers to the regular mode mentioned above.

We further investigate the spectral characteristic of the power splitter with focus on the case of equal splitting. For $f=5.6 \mathrm{GHz}$, the equal splitting is reached in the two 

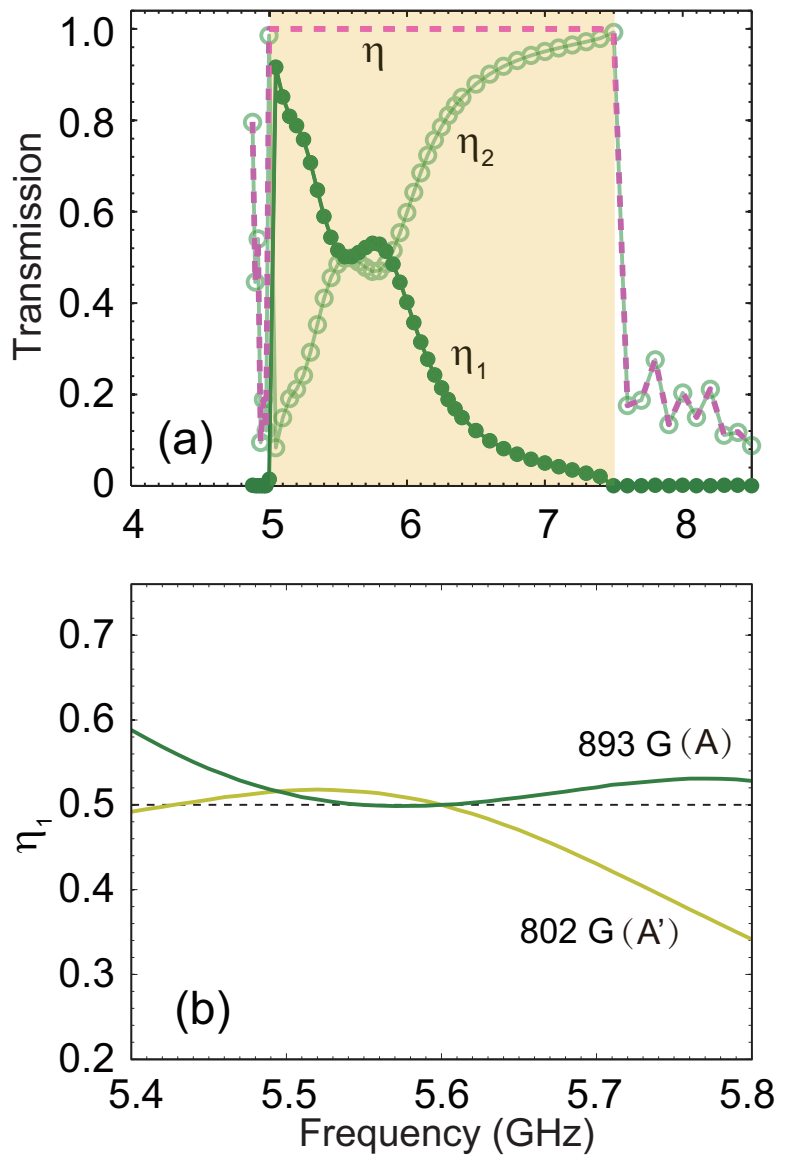

Figure 4. (Color online) (a) $\eta_{1}, \eta_{2}$ and $\eta=\left(\eta_{1}+\eta_{2}\right)$ as a function of $f$ for $\mathrm{H}_{0}=893$ G. (b) Transmission spectra in a frequency interval around $5.6 \mathrm{GHz}$ for $\mathrm{H}_{0}=893 \mathrm{G}$ and $802 \mathrm{G}$.

cases of $\mathrm{H}_{0}=893 \mathrm{G}$ and $802 \mathrm{G}$, which are marked by A and $\mathrm{A}^{\prime}$ in Fig. 3, respectively. Figure 4(a) shows the spectra of $\eta_{1}, \eta_{2}$, and $\eta\left(=\eta_{1}+\eta_{2}\right)$ for $\mathrm{H}_{0}=893 \mathrm{G}$. Both $\eta_{1}$ and $\eta_{2}$ vary largely with $f$, but $\eta$ is kept to be unity over the range $[5,7.5] \mathrm{GHz}$, which is exactly associated with the ROWP band for the YIG-air-metal waveguide under $\mathrm{H}_{0}=893 \mathrm{G}$. The equal splitting's band, defined as within $2 \%$ error for a desired splitting ratio, is $[5.51,5.65] \mathrm{GHz}$, as shown in Fig. 4(b). The $\eta_{1}$ spectrum for $\mathrm{H}_{0}=802 \mathrm{G}$ (marked by $\mathrm{A}^{\prime}$ ) is also calculated and presented in Fig. 4(b) for comparison. The spectra for the two cases are rather different, and the one for $\mathrm{H}_{0}=893 \mathrm{G}$ has a relatively large bandwidth because the derivative $d \eta_{1} / d f$ vanishes at $f=5.6 \mathrm{GHz}$. Note that the derivative $d \eta_{1} / d H_{0}$ also vanishes at $\mathrm{H}_{0}=893$ for $f=5.6 \mathrm{GHz}$, as seen in Fig. 3(a). Evidently, $\eta_{1}$ as functions of both $f$ and $\mathrm{H}_{0}$ possesses an equilibrium point at $f=5.6$ $\mathrm{GHz}$ and $\mathrm{H}_{0}=893 \mathrm{G}$, where $\eta_{1} / \eta_{2}=0.5 / 0.5$. This equilibrium point is beneficial to get a large bandwidth for the equal splitting.

The existence of the equilibrium point provides a promising way to achieve larger bandwidth for desired splitting ratio. For our proposed high-efficiency splitter, the equilibrium point (at a certain frequency) can also be attained for any unequal splitting 

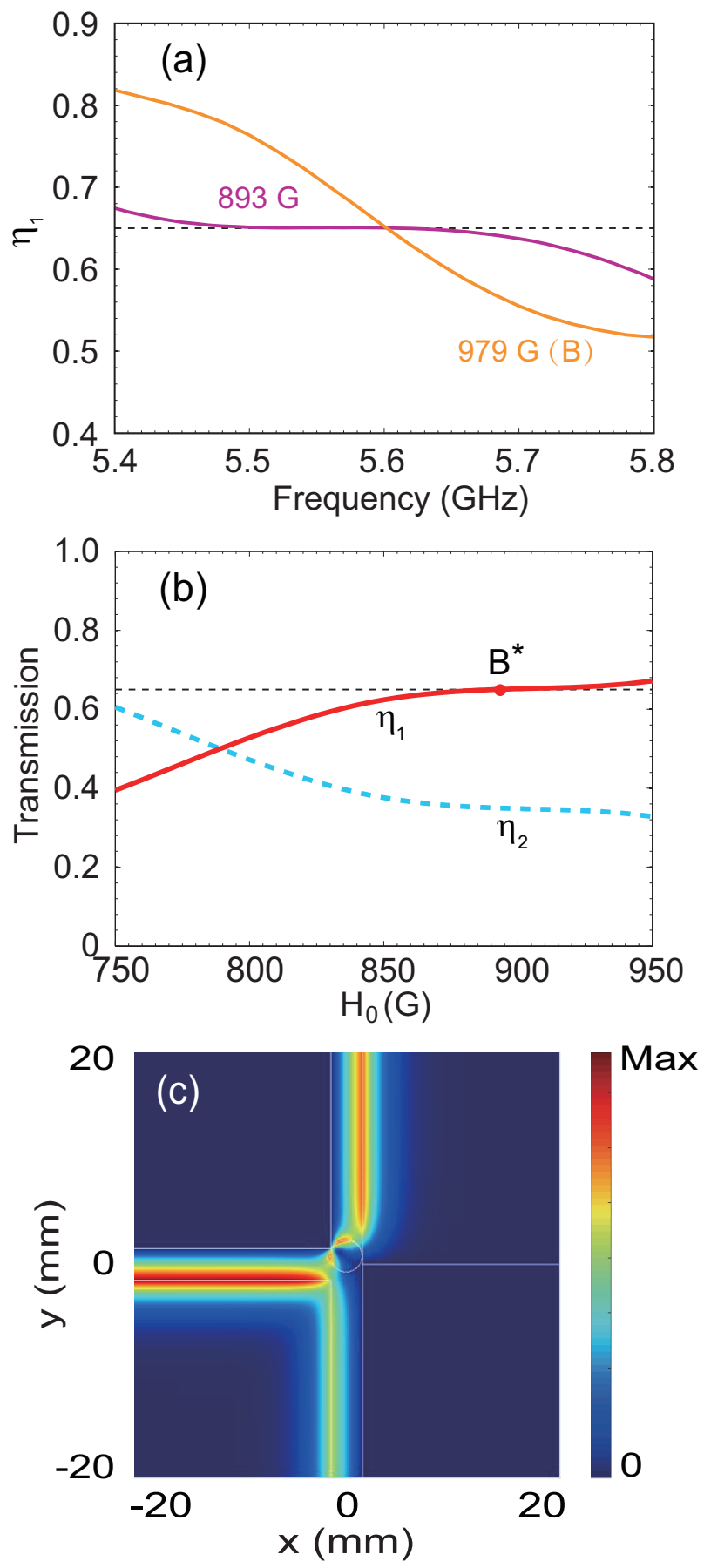

Figure 5. (Color online)(a) $\eta_{1}$ as a function of $f$ for two unequal power splitters. The YIG rod is centered at $\mathrm{C}(-0.05,0.80) \mathrm{mm}$ for $\mathrm{H}_{0}=893 \mathrm{G}$ and at $\mathrm{C}(0,1.03) \mathrm{mm}$ for $\mathrm{H}_{0}=979 \mathrm{G}$. For both the splitters, $\eta_{1} / \eta_{2}=0.65 / 0.35$ at $f=5.6 \mathrm{GHz}$. (b) $\eta_{1}$ and $\eta_{2}$ as functions of $\mathrm{H}_{0}$ for $f=5.6 \mathrm{GHz}$. The YIG rod is located at $\mathrm{C}(-0.05,0.80) \mathrm{mm}$. (c) Simulated $\mathbf{E}$ amplitudes for the equilibrium point of $\mathrm{H}_{0}=893 \mathrm{G}$ and $f=5.6 \mathrm{GHz}$ when the YIG rod is centered at $\mathrm{C}(-0.05,0.80) \mathrm{mm}$.

by adjusting the position of the YIG rod. To show this, we consider an example of unequal splitting with $\eta_{1}=0.65$ and $\eta_{2}=0.35$. As seen from Fig. 3(a), this splitting 
behaviour happens at $f=5.6 \mathrm{GHz}$ and $\mathrm{H}_{0}=979 \mathrm{G}$ (marked by $\mathrm{B}$ ), and obviously, it is not an equilibrium point for $\eta_{1} / \eta_{2}=0.65 / 0.35$, because $d \eta_{1} / d H_{0} \neq 0$ at $\mathrm{H}_{0}=979 \mathrm{G}$. In this case, it is found that $d \eta_{1} / d f$ also does not vanish at $f=5.6 \mathrm{GHz}$, as shown in Fig. $5(\mathrm{a})$, where the $\eta_{1}$ spectrum is plotted for $\mathrm{H}_{0}=979 \mathrm{G}$. For $f=5.6 \mathrm{GHz}$, we analyze the dependence of $\eta_{1}$ on $\mathrm{H}_{0}$ for different YIG-rod positions, and find that $d \eta_{1} / d H_{0}=0$, see in Fig. 5(b), appears at $\mathrm{H}_{0}=893 \mathrm{G}$ when $x_{c}=-0.05 \mathrm{~mm}$ and $y_{c}=0.8 \mathrm{~mm}$ (marked by $\mathrm{B}^{*}$ ). The $\eta_{1}$ spectrum for $\mathrm{H}_{0}=893$ is also displayed in Fig. 5(a), and as expected, $d \eta_{1} / d f$ vanishes at $f=5.6 \mathrm{GHz}$ as well. Hence, the point of $f=5.6 \mathrm{GHz}$ and $\mathrm{H}_{0}=893 \mathrm{G}$ is an equilibrium point for $\eta_{1} / \eta_{2}=0.65 / 0.35$. But this equilibrium point is rather different from that (marked by A) in Fig. 3(a) in mathematics, the latter is a inflection point for the transmission coefficient $\eta_{1}$ (or $\eta_{2}$ ) as the function of $f$ and $\mathrm{H}_{0}$. Clearly, the present equilibrium point marked by $\mathrm{B}^{*}$ in Fig. $5(\mathrm{~b})$ is a stationary point, where $\partial \eta_{1} / \partial f$ and $\partial \eta_{1} / \partial H_{0}$ also vanish. In contrast, the point $\mathrm{B}$ in Fig. 3(a) is not an equilibrium point, because $\partial \eta_{1} / \partial H_{0}$ as well as $\partial \eta_{1} / \partial f$ are nonzero. Evidently, there exist at least two kinds of equilibrium point for $\eta_{1}$ (or $\left.\eta_{2}\right)$, and this increases the possibility of the existence of equilibrium point for arbitrary splitting ratio. By searching for the equilibrium point, we here obtain a relatively larger bandwidth for the unequal splitting of $\eta_{1}=0.65$ and $\eta_{2}=0.35$. The large bandwidth for a desired splitting ratio (under a certain external magnetic field) is of importance for pulse signal processing. Figure 5(c) shows the simulated electric amplitudes for the equilibrium point of $f=5.6$ $\mathrm{GHz}$ and $\mathrm{H}_{0}=893 \mathrm{G}$. The YIG rod is located at $\mathrm{C}(-0.05,0.80) \mathrm{mm}$. As expected, the field amplitudes in the upper output channel are apparently stronger than those in the lower one, which is obviously different from the case of equal splitting in Fig. 3(c).

If signals to deal with are monochrome or quasi-monochrome, large operation range can be easily obtained for our proposed splitter by tuning the external magnetic field. Evidently, in this situation, the operation range for a certain splitting ratio is only determined by the ROWP behaviour of the input channel. The value of $\mathrm{H}_{0}$ versus frequency for $\eta_{1} / \eta_{2}=0.5 / 0.5$ is evaluated and plotted as the solid line in Fig. 6, where $x_{c}=-0.05 \mathrm{~mm} y_{c}=0.8 \mathrm{~mm}$, and the other parameters of the system are the same as in Fig. 1. The operation range is from $4 \mathrm{GHz}$ to $7 \mathrm{GHz}$, while $\mathrm{H}_{0}$ is tuned from $277 \mathrm{G}$ to $1265 \mathrm{G}$. One-way propagation is also available for the whole frequency range. More interestingly, the value of $\mathrm{H}_{0}$ almost increases linearly with the frequency in the range $[4,7] \mathrm{GHz}$, as shown in Fig. 6. This situation also happens for other splitting ratio. To show this, another example of unequal splitting with $\eta_{1}=0.65$ and $\eta_{2}=0.35$ is also displayed as the dashed line in Fig. 6, where $x_{c}=0 \mathrm{~mm} y_{c}=1.03 \mathrm{~mm}$. Here the operation band ranges from $4 \mathrm{GHz}$ to $7 \mathrm{GHz}$, when $\mathrm{H}_{0}$ is tuned from $443 \mathrm{G}$ to $1450 \mathrm{G}$. In the region $[4,7] \mathrm{GHz}, \mathrm{H}_{0}$ versus $f$ for $\eta_{1} / \eta_{2}=0.65 / 0.35$ is similar to the first case as expected. For both examples, the transmission efficiency of $100 \%$ is preserved. 


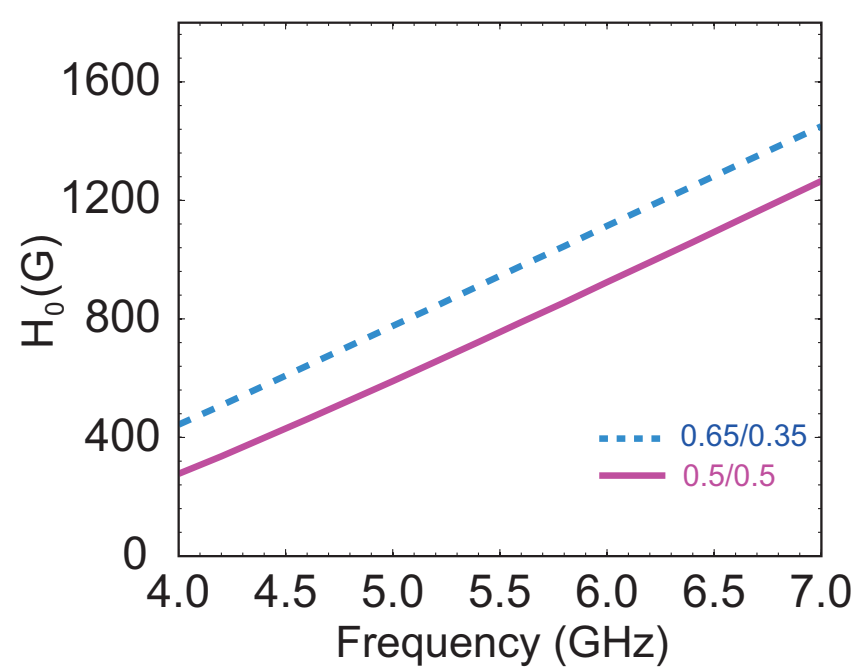

Figure 6. (Color online) Splitting ratio versus $\mathrm{H}_{0}$ and $f$ for the proposed splitters. The solid and dashed lines represent the equal splitting and unequal splitting with $\eta_{1} / \eta_{2}=0.65 / 0.35$, respectively. The YIG rod lies at $\mathrm{C}(-0.05,0.80) \mathrm{mm}$ for $\eta_{1} / \eta_{2}=0.5 / 0.5$ and at $\mathrm{C}(0,1.03) \mathrm{mm}$ for $\eta_{1} / \eta_{2}=0.65 / 0.35$. The other parameters are the same as in Fig. 1.

\section{Conclusions}

We have proposed and studied novel tunable T-shaped splitters utilizing one-way waveguide, where the one-way waveguide is built on the YIG-air-metal configuration with one static magnetic field applied. The guiding properties of the YIG-dielectricmetal structure has been analyzed, and the waveguide exhibits a robust one-way propagation over a large frequency range. The transmission efficiency of the splitter is $100 \%$ over the ROWP range provided that the materials in the system are lossless. When introducing the defect in the junction, the splitting ratio is strongly modified due to the asymmetric feature of two output channels. Our results further illustrate that the splitting ratio of the splitter can be gradually tuned by varying the applied magnetic field. The power splitter proposed here works on radio frequency in a two-dimensional configuration, and it can be easily extended to a three-dimensional structure and to the subject of integrated photonics.

\section{Acknowledgments}

National Natural Science Foundation of China (NSFC) (61372005); National Natural Science Foundation of China (NSFC) under a key project (41331070); Natural Science key project of the Higher Education Institutes of Anhui Province, China (KJ2016A759); Innovation Fund Designated for Graduate Students of Nanchang University, China(CX2017077). 


\section{References}

[1] Zhou Y J and Cui T J 2011 Applied Physics Letters 98221901

[2] Hwang R B, Hsu N C and Chin C Y 2012 IEEE Transactions on Antennas and Propagation 60 $417-420$

[3] Gao X, Hui Shi J, Shen X, Feng Ma H, Xiang Jiang W, Li L and Jun Cui T 2013 Applied Physics Letters 102151912

[4] Sugimoto Y, Ikeda N, Carlsson N, Asakawa K, Kawai N and Inoue K 2002 Optics letters 27 388-390

[5] Yu X and Fan S 2003 Applied Physics Letters 83 3251-3253

[6] Huang J, Yang J, Chen D, He X, Han Y, Zhang J and Zhang Z 2018 Photonics Research 6 574-578

[7] Chen X, Wen R D, Shi J L and Tseng S Y 2018 Journal of Optics 20045804

[8] Bozhevolnyi S I, Volkov V S, Devaux E, Laluet J Y and Ebbesen T W 2006 Nature 440508

[9] Suzuki K, Cong G, Tanizawa K, Kim S H, Ikeda K, Namiki S and Kawashima H 2015 Optics express 23 9086-9092

[10] Calmano T, Kränkel C and Huber G 2015 Optics letters 40 1753-1756

[11] Hanzawa N, Saitoh K, Sakamoto T, Matsui T, Tsujikawa K, Koshiba M and Yamamoto F 2013 Optics express 21 25752-25760

[12] Rouifed M S, Littlejohns C G, Tina G X, Qiu H, Penades J S, Nedeljkovic M, Zhang Z, Liu C, Thomson D J, Mashanovich G Z et al. 2017 Optics Express 25 10893-10900

[13] Tao S, Fang Q, Song J, Yu M, Lo G and Kwong D 2008 Optics express 16 21456-21461

[14] Fan S, Johnson S G, Joannopoulos J, Manolatou C and Haus H 2001 JOSA B 18 162-165

[15] Boscolo S, Midrio M and Krauss T 2002 Optics Letters 27 1001-1003

[16] Wilson R, Karle T J, Moerman I and Krauss T F 2003 Journal of Optics A: Pure and Applied Optics 5 S76

[17] Frandsen L H, Borel P I, Zhuang Y, Harpøth A, Thorhauge M, Kristensen M, Bogaerts W, Dumon $\mathrm{P}$, Baets R, Wiaux V et al. 2004 Optics letters 29 1623-1625

[18] Yang D, Tian H and Ji Y 2012 Optics Communications 285 3752-3757

[19] Zhang X, Li W and Jiang X 2012 Applied Physics Letters 100041108

[20] Wang Z, Shen L, Yu Z, Zhang X and Zheng X 2013 JOSA B 30 173-176

[21] Haldane F and Raghu S 2008 Physical review letters 100013904

[22] Raghu S and Haldane F 2008 Physical Review A 78033834

[23] Brion J, Wallis R, Hartstein A and Burstein E 1972 Physical Review Letters 281455

[24] Kuzmiak V, Eyderman S and Vanwolleghem M 2012 Physical Review B 86045403

[25] Yu Z, Veronis G, Wang Z and Fan S 2008 Physical review letters 100023902

[26] Wang Z, Chong Y, Joannopoulos J D and Soljačić M 2009 Nature 461772

[27] Hartstein A, Burstein E, Maradudin A, Brewer R and Wallis R 1973 Journal of Physics C: Solid State Physics 61266

[28] Shen L, You Y, Wang Z and Deng X 2015 Optics express 23 950-962

[29] Tsakmakidis K, Shen L, Schulz S, Zheng X, Upham J, Deng X, Altug H, Vakakis A and Boyd R 2017 Science 356 1260-1264

[30] He C, Chen X L, Lu M H, Li X F, Wan W W, Qian X S, Yin R C and Chen Y F 2010 Applied Physics Letters 96111111

[31] Deng X, Hong L, Zheng X and Shen L 2015 Applied optics 54 4608-4612

[32] Pozar D M 2009 Microwave engineering (John Wiley \& Sons) 